\title{
Erratum to: Social Capital and Subjective Well-Being in Japan
}

\author{
Midori Matsushima ${ }^{1} \cdot$ Yoshiho Matsunaga $^{1}$
}

Published online: 10 July 2015

(C) International Society for Third-Sector Research and The Johns Hopkins University 2015

\section{Erratum to: Voluntas DOI 10.1007/s11266-015-9581-3}

The following acknowledgement was inadvertently omitted from this paper.

Acknowledgment This research is funded by the Osaka University of Commerce Research Fund (from April 2012 to March 2014). We are grateful for the support from Osaka University of Commerce. We would also like to thank the JGSS Research Center at Osaka University of Commerce for providing the data. The Japanese General Social Surveys (JGSS) are designed and carried out by the JGSS Research Center at Osaka University of Commerce (Joint Usage / Research Center for Japanese General Social Surveys accredited by the Minister of Education, Culture, Sports, Science and Technology), in collaboration with the Institute of Social Science at the University of Tokyo.

Also, in the third line of the abstract, there is reference to: Japan General Survey 2010. This should be: Japan General Social Survey (JGSS) 2010.

The online version of the original article can be found under doi:10.1007/s11266-015-9581-3.

Midori Matsushima

midori@daishodai.ac.jp

1 Faculty of Business Administration, Osaka University of Commerce, 4-1-10 Mikuriyasakaemachi, Higashiosaka-shi 577-8505, Osaka, Japan 